\title{
AT1 Receptor Antagonists: Pharmacological Treatment of Hypertension in Brazil
}

\author{
Vanessa Pepeliascov ${ }^{1}$, Kleber de Magalhães Galvão ${ }^{1}$, Dones Cláudio Janz $\mathrm{Jr}^{2}$, Helen Dutra Leite ${ }^{3}$, \\ Felipe de Lara Janz ${ }^{3, *}$ \\ ${ }^{1}$ Bandeirante University, Sao Paulo, Brazil \\ ${ }^{2}$ Ponta Grossa StateUniversity, Ponta Grossa Brazil \\ ${ }^{3}$ Universityof São Paulo, Sao Paulo, Brazil \\ *Corresponding author: fljanz@usp.br
}

\begin{abstract}
High blood pressure (HBP) is a multifactorial disease that affects millions of people around the world and contributes to a large number of deaths due to acute myocardial infarction, stroke and chronic kidney disease. Its etiology remains inconclusive, but it is known that it arises of central and peripheral catecholaminergic dysfunction. Thus, cellular mechanisms are still under investigation. Its pathophysiology is characterized by an increase in systolic and diastolic blood pressure levels. The national and international guidelines for hypertension indicate that effective pharmacotherapy provides a control in blood pressure values and mortality/morbidityreduction. Classes of antihypertensive drugs available for clinical use are diuretics, beta-blockers, alpha-blockers, sympatholytic, calcium channel antagonists, angiotensin converting enzyme inhibitors and angiotensin receptor antagonists of angiotensin II (ARBs). ARBs (i.e.: candesartan, irbesartan, losartan, olmesartan, telmisartan and valsartan)represent current and often used drug class in Brazil.They have different molecular configurations with independent action mechanismsin angiotensin II AT1 receptor. The objective of this paper is to discuss the pathophysiology and pharmacotherapy of hypertension, emphasizing the antagonists of angiotensin II used in Brazil, since they constitute a class of antihypertensive drugs that has fewer side effects and greater therapeutic efficacy.
\end{abstract}

Keywords: high blood pressure, pharmacotherapy, angiotensin II antagonists

Cite This Article: Vanessa Pepeliascov, Kleber de Magalhães Galvão, Dones Cláudio Janz Jr, Helen Dutra Leite, and Felipe de Lara Janz, "AT1 Receptor Antagonists: Pharmacological Treatment of Hypertension in Brazil.” Biomedical Science and Engineering, vol. 3, no. 2 (2015): 41-43. doi: 10.12691/bse-3-2-3.

\section{Introduction}

\subsection{Blood Pressure and Control Mechanisms}

Peripheral vascular resistance (PVR) and cardiac output (CO) determine blood pressure (BP). Control of BP involves numerous substances and physiological mechanisms that interact to maintain the PA at appropriate levels in different situations. Control mechanisms result from the interaction of various physiological systems as cardiovascular, renal, neural and endocrine systems. The autonomic nervous system and its different hormonal types stand out in controlling blood pressure [1]. Dysfunctions in these control mechanisms, with consequent changes in the variables PVR and DC result in systemic hypertension (SH) [2]. Autonomic nervous system (ANS) plays an important role in controlling blood pressure.

According to the VI Brazilian Guidelines on Arterial Hypertension (2010) in 2007, there were 1,157,509 cases for cardiovascular disease (CVD) in the National Health System. With regard to costs, in November 2009 there were 91,970 hospitalizations for CVD, resulting in a cost of R\$165,461,644.33. End-stage renal disease, another condition often associated to hypertension, resulted in the inclusion of 94282 individuals undergoing dialysis in SUS(Health UnicSystem and 9,486 deaths in 2007.

We can classify this regulation in two ways: shorttermneuro-humoral regulation and medium and long-term regulation through the activation or inhibition of hormone systems or for retention and excretion of sodium [3]. ANS influences tonic and reflex cardiovascular system. Largely the effects caused by the nervous system in the cardiovascular system are due tothe action of neurotransmitters noradrenaline (NA) and acetylcholine (Ach). However, catecholamines release in other postganglionic sympathetic and parasympathetic terminals potentiate or decrease the activity of NA and Ach [4]. According to Guyton and Hall, 2002, [5] there are reflex mechanisms from the autonomic nervous system, that control the BP, the main ones are the baroreceptors, arterial chemoreceptors and lung receptors.

The blood pressure control by the baroreflex system (mediated by stimulation of baroreceptors aortic and carotid, called high-pressure receptors) acts in situations where it is required to keep track of blood pressure, such as physical exercise, postural changes or pathological situations like hemorrhage and hypothermia [6]. Arterial chemoreceptors are sensitive cells that monitor the amount of oxygen, carbon dioxide, and hydrogen ions in the blood. When BP falls below a critical level, chemoreceptors are 
stimulated by decreasing oxygen, carbon dioxide formation and hydrogen ions [7].

Baroreceptors are located in strategic positions between the heart and the brain, and chemoreceptors are located in the carotid bodies of two common carotid arteries and aortic bodies of the aorta [8]. Release of adrenaline and noradrenaline from the adrenal medulla, release of vasopressin by the neurohypophysis and the increase in plasma renin levels occur when sustained BP falls. These humoral mechanisms may performa cardiovascular response for minutes or even hours [6].

\subsubsection{Renin-angiotensin-aldosterone}

Renin-angiotensin-aldosterone system (RAAS) has been studied for over a century and found to play an important role in blood pressure regulation. This system helps to maintain cardiovascular homeostasis, control of volume and peripheral vascular resistance. Misfits in this system are directly involved in the onset of hypertension [9].

Activation of this system is given by prorenin, which is secreted in juxtaglomerular cells located in nephrons in response to an increase in sympathetic nervous system activity or a decrease in blood pressure, the volume of extracellular fluids and the extracellular sodium concentration [10]. Prorenin is then converted to renin. Three predominant mechanisms control the secretion of renin, which are macula dense, renal baroreceptor and sympathetic stimulation [9]. In the bloodstream, renin acts enzymatically in the conversion of angiotensinogen, which is a plasma protein produced in the liver and has 452 amino acids. Angiotensinogen is converted to angiotensin I, which is then converted to angiotensin. This conversion occurs in the passage of blood through the lungs through angiotensin-converting enzyme (ACE). ACE is composed of 1278 amino acids consisting of two domains: one amino terminal and one carboxyterminal. It is most frequently found on the luminal surface of vascular endothelial cells, also found in smooth muscle cells of the vessels, cardiomyocytes, proximal convoluted tubule kidney and brain tissue. Recently identified a homologue of ACE, ACE2 that critically regulates heart function, and provides a counteraction to the ACE [11].

There are 02 types of angiotensin II receptors, AT1 and AT2.The main actions of angiotensin II (ANG II) are mediated by these two receptors, especially in the control of blood pressure. Angiotensin II receptor blockers are highly selective to AT1 receptor. They act antagonistically, blocking the actions of ANG II. The AT1 receptor blockade results in inhibition of vasoconstriction and decreased aldosterone synthesis [12]. ANG II is a potent vasoconstrictor that acts on the short and long-term regulation of blood pressure. ANG II present in circulation reduces the excretion of sodium by increasing the absorption thereof in the proximal tubules of the kidney. The stimulation of aldosterone secretion by the adrenal gland that is responsible for increasing the retention of salt and water by the kidneys is mediated by ANG II, thus contributing to AP long-term regulation [13].

The AT1 and AT2 receptors membrane mediates the biological activity of ANG II. AT1 receptor promotes most of the known actions of ANG II effects such as vasodilation, inhibition of apoptosis and cell growth and differentiation, through the intermediate pathways involving substances such as diacylglycerol, inositol triphosphate, phospholipase $\mathrm{C}$ and calcium. AT2 receptors have a probable action in modulating the effects of angiotensin II to the AT1 receptor [10].

\section{Pharmacotherapy of Systemic Hypertension}

According to the Brazilian Hypertension Guidelines of 2010, the goals of pharmacological treatment of hypertension should be: reduction of morbidity and mortality in cardiovascular events and achievement of the goals set in pressure levels. Below is the classification of drugs for the treatment of hypertension:

a) Diuretics;

b) Centrally acting adrenergic inhibitors - alpha-2 central agonists;

c) Beta-blockers;

d) Alpha-blockers - alpha-1 adrenergic blockers;

e) Direct vasodilators;

f) Calcium channel blockers;

g) Angiotensin converting enzyme inhibitors;

h) AT1 receptor blockers of angiotensin II;

i) Direct renin inhibitor.

Studies have demonstrated reduction of morbidity and mortality in greater numbers with diuretics, beta-blockers, angiotensin converting enzyme inhibitors of angiotensin AT1 receptor blockers and calcium channel blockers. Among the therapeutic classes mentioned, the AT1 receptor blockers have shown greater efficacy in reducing cardiovascular morbidity and mortality (Ribeiro, 2008). The LIFE study (Losartan Intervention for The End Point Reduction in Hypertension Study), VALUE (Valsartan Antihypertensive Long-term Use Evaluation) and SCOPE (Study on Cognition in the Elderly) show comparable values in the reduction in blood pressure compared with the effects and classes cardio protective that provide better results than other therapies [11].

\subsection{Antagonists of Angiotensin II Receptor}

The arrival of antagonists of angiotensin II receptor in the pharmaceutical market is relatively recent. It began in the late 1980swith losartan and currently has several other representatives who appeared in the pharmaceutical market a few years ago. In Brazil, the marketed drugs are candesartan, irbesartan, losartan, olmesartan, telmisartan and valsartan.

Table 1. Angiotensin type II receptor blockers

\begin{tabular}{|c|c|}
\hline Candesartan & $8 \mathrm{mg}$ and $32 \mathrm{mg}$ \\
\hline Irbersartan & $150 \mathrm{mg}$ and $300 \mathrm{mg}$ \\
\hline losartan & $25 \mathrm{mg} 100 \mathrm{mg}$ \\
\hline Olmesartan & $20 \mathrm{mg}$ and $40 \mathrm{mg}$ \\
\hline Telmisartan & $40 \mathrm{mg}$ and $160 \mathrm{mg}$ \\
\hline Valsartan & 80 mgand $320 \mathrm{mg}$ \\
\hline
\end{tabular}

This antihypertensive drug class is relatively new, but has shown to reduce cardiovascular events related to hypertension. The first drug of this class was losartan. In Brazil, there are six types of angiotensin II receptor blockers (ARBs): losartan, valsartan, candesartan, telmisartan, olmesartan and ibersartan. LIFE, VALUE and 
SCOPE studies were conducted with hypertensive patients to verify the effectiveness of these ARBs. The LIFE study compared ARBs with atenolol and losartan; their results showed that losartan achieved a blood pressure reduction of 30.2 / 16.6 while atenololprovided a reduction of 29.1/16.8 mm/hg. The most significant values wereobtained in the reduction of cardiovascular events, with $25 \%$ reduction of cerebral vascular accident (CVA) and onset of diabetes, with treatment with losartan [14].

Beneficial effects of ARBs are primarily attributed to its ability to block specificallythe AT1 angiotensin II receptors, preventing its harmful effects. They have different molecular configurations that promote actions regardless ofthe form of block angiotensin II AT1 receptor. Among them, olmesartan is the one that has demonstrated greater reduction in blood pressure values. Olmesartan medoxomil is the newest drug of the class of AT1 receptor blockers of angiotensin II (ARBs). In comparative studies, it was effective in reducing blood pressure values higher than others in this class and similar to amlodipine [12]. ARBs, in general, act by blocking the action of the AT1 receptor, which is responsible for most actions hitherto known angiotensin II. Through the intermediate pathways involving substances such as diacylglycerol (DAG), inositol triphosphate (PI3), phospholipase C (PLC) and calcium, Angiotensin II also stimulates vasoconstriction promotes intracellular protein synthesis [10].

The roles of AT2 receptors are not well defined. They play a role of importance in fetuses and in adults in response to injury. It is believed that the AT2 receptor is involved in modulating the effects of angiotensin II to the AT1 receptor (counteracting the pro-synthesis stimulus) [9]. The antihypertensive effect of ARBs is comparable to other antihypertensive agents such as ACE inhibitors, calcium antagonists and beta-blockers. Irbesartan (300mg/day), candesartan (16 mg/day) appear to have antihypertensive effect more pronounced than losartan (100 mg / day to $50 \mathrm{mg} /$ day, respectively) in patients with essential hypertension [12]. ARBs result in an equal pressure drop that is indifferent to increasing the dose. This is a dose-response "shallow" curve when it comes to olmesartan, however, the curve has a steeper slope. Studies with olmesartan demonstrate that this molecule has the greatest number of functional sites (seven), and the hydrogen bonding and hydrophobic interactions provided by the hydroxyl radicals and alpha-carboxyl increase affinity for the AT1 receptor [15].

\subsubsection{Pharmacodynamics}

Angiotensin II receptor blockers (ARBs) are a class of antihypertensive drugs that block the RAAS, selectively antagonizing the ANG II receptor type AT1. ARBs can operate in dual manner, namely blocking the actions of ANG II by the antagonism of AT1 receptors and AT2 receptors stimulating by elevated circulating levels of ANG II [15]. The pharmacologically ARBs, have pharmacodynamics differences in the mechanism of action. However, generally the blockade of AT1 receptors, antagonizing the effects of Ang II, [13].

The basic mechanism of action of angiotensin II antagonists is to antagonize specifically the AT1 receptor, emptyingthe AT2 receptor. Angiotensin II antagonists have a different pharmacodynamics profile from ACE inhibitors; they do not block the converting enzyme and, thus, do not increase levels of bradykinin [8]. As stated within the ARBs classes there pharmacodynamics differences as we see below: Losartan binds selectively to the AT1 receptor and does not bind to or block other hormone receptors or ion channels that are important in cardiovascular regulation. Losartan is a potent orally active synthetic compound.Both in vitro and in vivo, losartan as a pharmacologically active metabolite carboxylic acid (E-3174) block all physiologically relevant actions of angiotensin II, regardless of the source or route of synthesis [16].

Candesartan cilexetil has a different mechanism of action when compared to losartan;the prodrug is rapidly converted to the active drug, candesartan, by ester hydrolysis during absorption in the gastrointestinal tract. Candesartan is an antagonist of angiotensin II receptor, selective for AT1 receptors, with tight binding to it and slow dissociation from the receptor. Candesartan does not bind to or block other hormone receptors or ion channels known to be important in cardiovascular regulation [17].

Olmesartan medoxomil is a prodrug as well as candesartan. During absorption from the gastrointestinal tract, it is hydrolyzed to olmesartan, the biologically active compound. It is a selective antagonist of angiotensin II receptor subtype AT1. Olmesartan binds competitively and selectively to the AT1 receptor, preventing the vasoconstrictor effects of ANG II selectively blocking its binding to the AT1 receptor in vascular smooth muscle. Its action does not depend on the route of synthesis of ANG II [18].

Telmisartan removes, with very high affinity, angiotensin II from its binding sites at the AT1 receptor, which is responsible for the known actions of angiotensin II. Telmisartan has no partial agonist activity at the AT1 receptor selectively and binds to these receptors. This connection is long lasting. Telmisartan does not show affinity for other receptors, including AT2 [19]. Valsartan is a potent and specific antagonist of Ang II receptor active orally. It acts selectively on the AT1 receptor subtype. Increased plasma levels of Ang II according to the AT1 receptor blockade with valsartan may stimulate the unblocked AT2 receptor, which appears to counterbalance the effect of the AT1 receptor [20].

\subsubsection{Pharmacokinetics}

ARBs have low oral bioavailability, usually lower than $50 \%$, except for irbesartan (70\%) and high plasma protein binding (>90\%). Most ARBs are metabolized by the liver and therefore should be used with caution in patients with impaired liver function, especially for telmisartan. Age, ethnicity and sex do not seem to interfere with the efficacy of these drugs [21].

Oral losartan is well absorbed and undergoes first-pass metabolism, forming an active carboxylic acid metabolite and other inactive metabolites. Presents a systemic bioavailability of approximately 33\%, achieving its mean maximum concentration, of its active metabolite in 1 hour and in 3 to 4 hours, respectively.Losartan and its active metabolite present the plasma protein binding rate $>99 \%$, mainly to albumin. The volume of distribution of losartan is 34 liters. Studies in rats indicate that losartan does not substantially cross the blood-brain barrier. After oral administration, plasma concentrations of losartan and its active metabolite exponentially decrease with terminal 
half-life of approximately 2 hours and 6 to 9 hours, respectively. During the administration of a single daily dose of $100 \mathrm{mg}$ losartan and its active metabolite do not accumulate in plasma. Biliary and urinary excretion contribute to the elimination of losartan and its metabolites [22].

Olmesartan medoxomil is described chemically as two, 3-diidroxi-2-butenil-4-(1-hidroxi-1-metiletil)-2-propil-1[p-(o-1H-tetrazol-5fenil) benzil] imidazol-5-carboxilato, 2, 3-cycliccarbonate. The empirical formula is C29H30N606. Its bioactivation by ester hydrolysis occurs to olmesartan during absorption from the gastrointestinal tract. Appears to be eliminated in a biphasic fashion, with a half-life of 615 hours. Pharmacokinetics of olmesartan is linear following single oral doses and multiple oral doses higher than therapeutic doses. Olmesartan binds competitively and selectively to the AT1 receptor, the action is independent of the synthesis pathways of angiotensin II [23]. The absolute bioavailability is of approximately $26 \%$. The maximum plasma concentration (Cmax) after oral administration is reached after approximately 2 hours. Approximately $30 \%$ to $50 \%$ of the absorbed dose is recovered in the urine, while feces excrete the remainder.

The prodrug candesartan cilexetil is converted to the active drug candesartan. The absolute bioavailability of candesartan is about $40 \%$ after an oral solution of candesartan cilexetil. The relative bioavailability of candesartan cilexetil tablets, compared with the same oral solution is approximately 34\%, with very little variability. The mean peak plasma concentration (Cmax) occurs 3-4 hours after ingestion of the tablet. Candesartan is highly bound to plasma proteins (> 99\%). Candesartan is eliminated primarily unchanged by urinary tract and bile and only a small part is eliminated by hepatic metabolism [24].

Telmisartan possesses rapid absorption, but the amount absorbed varies. The mean absolute bioavailability is about $50 \%$. Telmisartan predominantly binds to plasma proteins (99.5\%), mainly albumin and alpha-1 acid glycoprotein. The metabolism takes place by conjugation to glucuronide. Telmisartan is characterized by a biexponential decay kinetics with a terminal half-life greater than 20 hours. Cumulative urinary excretion is less than $2 \%$ of the dose.

Valsartan after oral administration exhibits a mean absolute bioavailability of $23 \%$. Valsartan has an exponential decay kinetics (t1/2 alpha $<1 \mathrm{~h}$ and $\mathrm{t} 1 / 2$ beta about $9 \mathrm{am}$ ). The pharmacokinetics of valsartan is linear in the tested dose range. No changes occur in the kinetics of valsartan on repeated administration and there is little accumulation when administered once daily. The binding rate to serum proteins (94-97\%), mainly serum albumin. Of the total absorbed dose, $70 \%$ are excreted in feces and $30 \%$ in the urine, mainly as unchanged drug [24].

\section{Conclusion}

Based on the literature review we concluded that hypertension is a disease that affects a significant portion of the world population. In the literature there is no consensus regarding the etiology of hypertension. Thus, the molecular and cellular mechanisms involved in the pathophysiology remain under investigation. However, it is known that there is a central and peripheral catecholamine dysfunction in arterial hypertension. Scientists are still searching for a drug therapy that is effective in the treatment of hypertension, as well as providing obtaining the control target, a reduction in cardiovascular morbidity and mortality. Currently, there are several classes of drugs for hypertension that present varying mechanisms of action. Among these classes of drugs, the AT1 receptor blockers angiotensin I, have proven to be effective in treating this pathology by reducing the rates of cardiovascular mortality and morbidity. ARBs have among themselves in different ways AT1 receptor blocking action, due to its molecular differences. Olmesartan medoxomil is the newest drug of this class andprovides greater reduction in blood pressure. The side effects of ARBs are comparable to placebo. Thus, there is a greater patient compliance to treatment and a positive response in the treatment of hypertension.

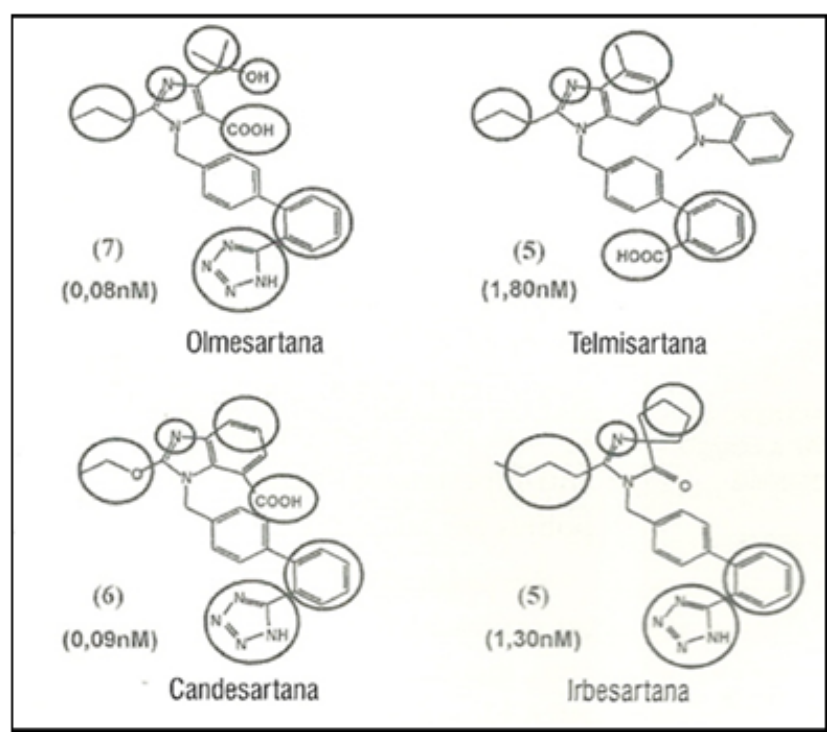

Figure 1. Molecular structure of main antagonists of the angiotensin II in Brazil

\section{References}

[1] Cesaretti, Mario Luis Riberio; Ginoza, Milton; Kohimann Junior, Osvaldo. Neural mechanisms: sympathetic nervous system. In: Ribeiro, Artur Beltrame; Plavnik, Frida Liane. Update on clinical hypertension, diagnostic and therapeutic: Pathophysiology of hypertension. London: Atheneu, 2008. Chapter 9, p. 79-85.

[2] Krieger, Eduardo Moacyr; Irigoyen, Maria Claudia; Krieger, José Eduardo. Pathophysiology of hypertension. Journal of Cardiolologia Society of the State of São Paulo, São Paulo, P.01-7, 1999.

[3] Ribeiro, José Márcio; Florencio Leonardo P.Pharmacological blockade of the renin-angiotensin-aldosterone system: converting enzyme inhibition and AT1 receptor antagonism. Brazilian Journal of Hypertension, Sao Paulo, n., P.293-302, 2000.

[4] PR Vade Mecum. Vade Mecum medications. RGR 17 publications ed. São Paulo, 2011.

[5] Rang, H.P; Dale, M.M, Pharmacology, 3rd ed. Publisher Koogan Guanabara, Rio de Janeiro, 1997.

[6] Guyton, AC, HallL, JE Treaty of Medical Physiology 10. Ed. Rio de Janeiro Guanabara Koogan 2002.

[7] Cipullo, José Paulo et al. Prevalence and Risk Factors for Hypertension in a Brazilian Population Urbana. Brazilian Archives of Cardiology, Sao Paulo, P.520-526, 2010.

[8] Franklin, Stanley S. et al. Cardiovascular Morbidity and Mortality in Hypertensive Patients with Lower Risk Higher Versus LIFE Substudy. American Heart Association, Dallas, p.492-499, 2005. 
[9] Gomes, Marco Antonio Mota et al. Treatment of Hypertension with olmesartan medoxomil in scheduling. Brazilian Archives of Cardiology, Sao Paulo, p.185-193, 2008.

[10] Kreutz, R. Olmesartan / amlodipine: A review of its use in the management of hypertension. Vascular Health andRisk Management, Princeton, p. 183-192. 2011.

[11] Lopes, Heno Ferreira; GIL, Juliana Dos Santos; ConsolimColombo, Fernanda Marciano. Activation of the adrenergic system, renin-angiotensin-aldosterone, endothelin and adrenomedullin in hypertension. Journal of the Society of Cardiology of the State of São Paulo, São Paulo, v. 18, no. 2, p.102-107, 2008.

[12] Mion Jr, Decius et al. The importance of antihypertensive medication treatment adherence. Brazilian Journal of Hypertension, Rio de Janeiro, p.55-58, 2006.

[13] Neves, Francis ofAssisi; Campos, Alessandra Menezes. Reninangiotensin-aldosterone In: Ribeiro, Arthur Beltrame; Plavnik, Frida Liane Update on clinical hypertension, diagnosis and treatment. London: Atheneu, 2008.

[14] Oigman, Wille; Fritsche, Mario Toros. Drugs that intervene in the renin-angiotensin system. Brazilian Journal of Hypertension, Rio de Janeiro, v.5, p.84-90, 1998.

[15] Oparil, Suzanne et al. Comparative efficacy of olmesartan, losartan, valsartan and irbesartan in the control of primary hypertension. The jornaul of Clinical Hypertension, vol. 3, no. 5, p. 202-282, 2001

[16] Tamargo, Juan et al. Pharmacological characteristics of ARB them. Son all iguales? Spanish Journal of Cardiology, vol. 6, p 10-24, 2006.
[17] Trapp, Silvia Manduca, VailatiI, Maria do Carmo Fernandes, Matsubara, Beatriz Bojikian, Schwartz, Denise Saretta. Effects of angiotensin II in the cardiovascular system. Archives of Veterinary Science, North America, 14, jun. 2009.

[18] Verdecchia, Paolo et al. Comparative assessment of angiotensin receptor blockers in different clinical settings. Vascular Health and Risk Management, Princeton, n., P.939-949, November 2008.

[19] Rigatto, Katya Vianna, Bohlke, Maristela, Irigoyen, Maria Claudia. Renin angiotensin system: from physiology to therapy. Magazine Society of Cardiology of Rio Grande do Sul. 2004.

[20] Salgado, Wilson. Control of blood pressure to prevent cardiovascular disease. In: Santos, Raul D. Manual of prevention of cardiovascular diseases. London: Atha, 2008.

[21] Povoa, Rui; Bombig, Maria Teresa Nogueira. Hypertension secondarily renal and endocrine origin: diagnosis and treatment. Journal of Cardiolologia Society of the State of São Paulo, São Paulo, n., P.122-134, 2008.

[22] Consolim-Colombo, Fernanda M; Krieger, Eduardo Moacyr. Sympathetic nervous system and hypertension. Brazilian Hypertension magazine, Rio de Janeiro, v 11, p. 275-278, 2004.

[23] Correa, Thiago Domingos et al. Hypertension: an update on the epidemiology, diagnosis and treatment. Medical files Abc, Sao Paulo, n. 5, P.90-101, 2006.

[24] Brazilian Guidelines on Hypertension, Brazilian Archives of Cardiology, vol. 95, 2010.

[25] BRASIL. Ministério da Saúde. Cadernos de Atenção Básica: Hipertensão Arterial Sistêmica. n. 15. Brasília, 2006.Link: http://bvsms.saude.gov.br/bvs/publicacoes/caderno_atencao_basic a15.pdf. 